NASA Contractor Report 182285

AIAA-89-1744

\title{
Numerical Studies of the Effects of Jet-Induced Mixing on Liquid-Vapor Interface Condensation
}

\author{
(MASA-CB-182285) NOEERICAL STELIES OF THE \\ ERECTS OF JET-IBLUCEL EIXIIG C \\ LIOUID-VAPCB INTEEFACE COADERSATICA Final

Chin-Shun Lin

Analex Corporation

NASA Lewis Research Center

Cleveland, Ohio

April 1989

Prepared for

Lewis Research Center

Under Contract NAS3-24564 
NUMERICAL STUDIES OF THE EFFECTS OF JET-INDUCED MIXING ON

LIQUID-VAPOR INTERFACE CONDENSATION

\author{
Chin-Shun Lin \\ Analex Corporation \\ NASA Lewis Research Center \\ Cleveland, Ohio 44135
}

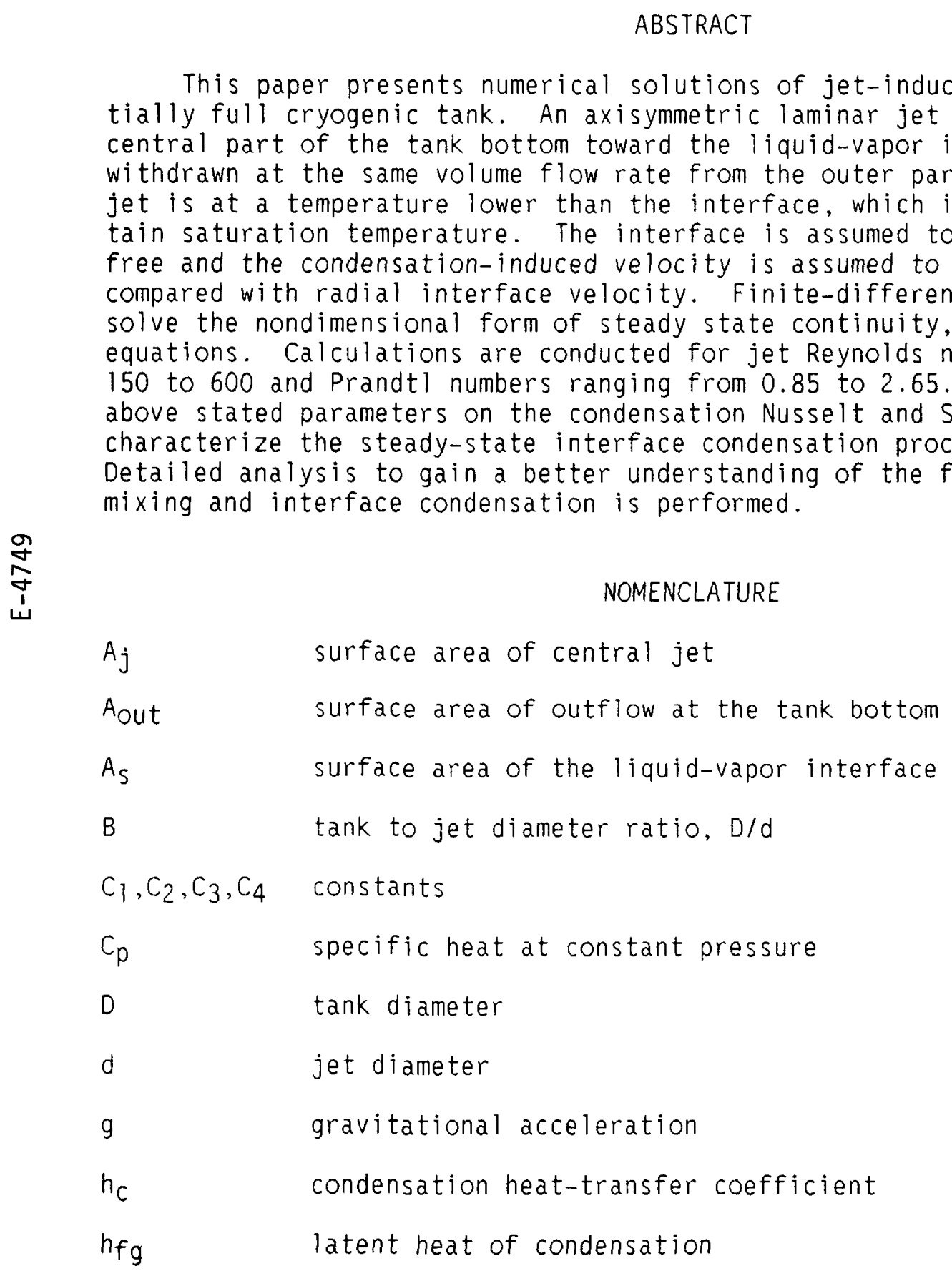




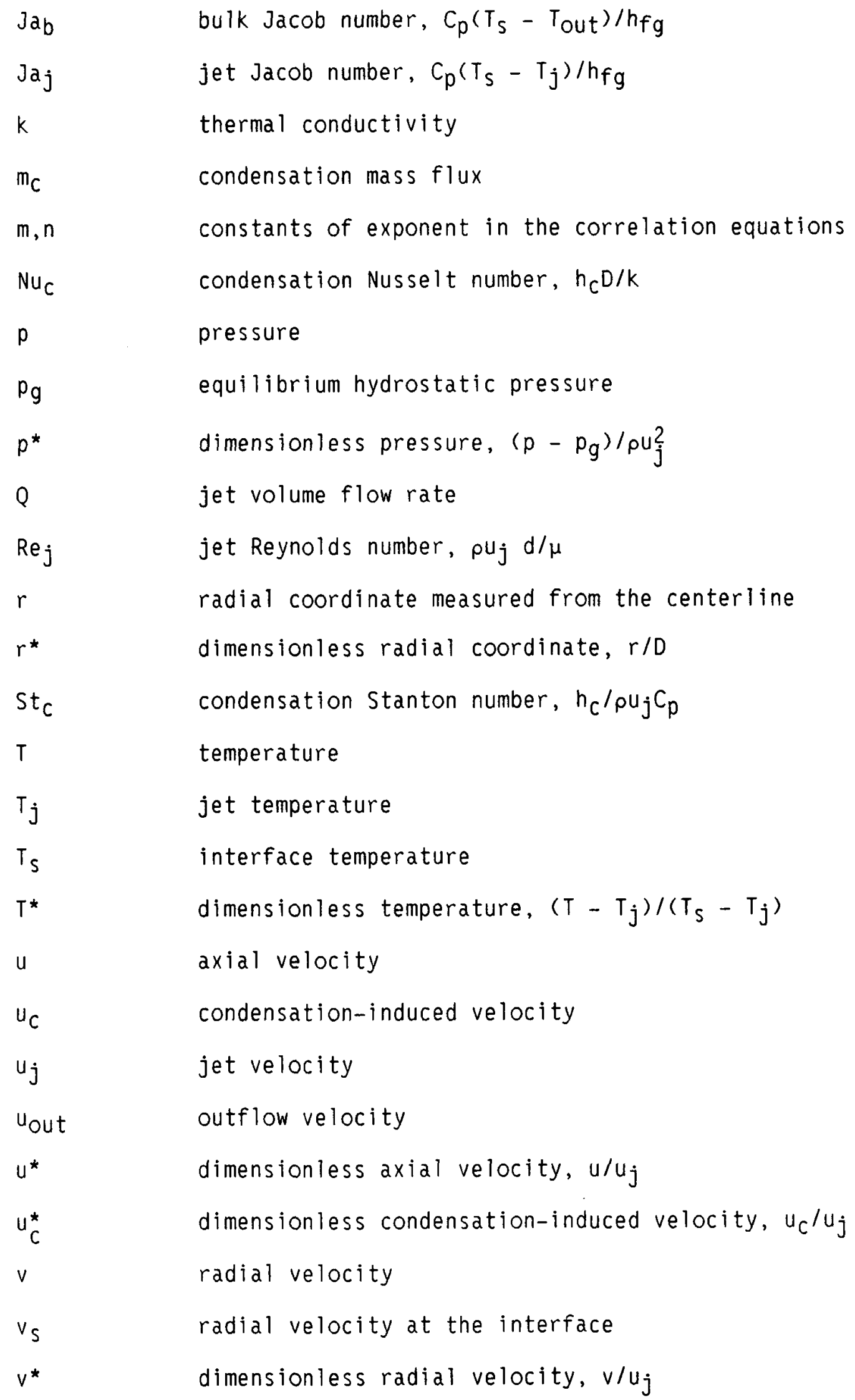




$\begin{array}{ll}v_{s}^{*} & \text { dimensionless radial velocity at interface } \\ x & \text { axial coordinate measured from the tank bottom } \\ x^{*} & \text { dimensionless axial coordinate, } x / D \\ x_{p} & \text { potential core length } \\ x_{s} & \text { liquid filling height }\end{array}$

Greek symbols:

$\begin{array}{ll}\mu & \text { dynamic viscosity } \\ \rho & \text { liquid density } \\ - & \text { average value over the interface }\end{array}$

Subscripts:

j

out

s

c

$c t$

b

\author{
evaluated at jet inlet \\ evaluated at outflow location \\ evaluated at liquid-vapor interface \\ evaluated at condensation condition \\ evaluated at tank centerline \\ evaluated at bulk liquid
}

\section{INTRODUCTION}

The thermal environment in space can result in radiation heat leak into the cryogen storage tanks and increase the tank pressure. Of the various technologies (ref. 1) being developed for tank pressure control, axial jet-induced mixing (ref. 2) is considered as an efficient method for a short-term storage system. The mixing of the tank contents causes a forced convection and removes heat quickly from the interface into the bulk liquid. The induced interface condensation then results in the reduction of tank pressure. It is obvious that the vapor condensation on the subcooled liquid at the interface plays a key role in reducing the tank pressure.

Several studies of steady-state fluid mixing and condensation rate have been conducted. Thomas (ref. 3) measured the condensation rate of steam on water surfaces mixed by a submerged jet. The liquid height to tank diameter ratio was varied from 0.32 to 1.3 . It was found that the condensation rate was roughly proportional to the jet Reynolds number. Dominick (ref. 4) conducted experiments to investigate the effects of jet injection angle and jet flow rate on the condensation rate in a Freon 113 tank. The ratio of liquid height to 
tank diameter was about one. The tests showed that heat transfer at the interface was enhanced by the mixing process as jet injection angle became more normal to the interface. The average heat transfer coefficient at the interface was found to be increasing with jet Reynolds number to a power of 0.73 . Sonin, Shimko, and Chun (ref. 5) also measured the steady-state condensation rate of steam in a water tank with liquid height to tank diameter ratio greater than three. The condensation process was dominated by the liquid-side turbulence near the interface. About 0.3 of tank diameter below the interface, the turbulence was found isotropic and essentially unaffected by the proximity of the surface. A correlation between the condensation rate and characteristic interface turbulent velocity was also developed in reference 5 . Hasan and Lin (ref. 6) used a finite-difference method to numerically solve time-averaged conservation equations along with $K-\varepsilon$ turbulence model for the prediction of turbulent velocity. Since the information about the turbulence at the interface was not available, zero gradients of $k$ and $\varepsilon$ were assumed for the boundary condition applied at the interface. The numerical prediction was in good agreement with Sonin's data except for the region close to the interface where diffusion process was dominant. The failure of numerical solution for the near-interface region, as stated in reference 6 , was probably due to the inappropriate turbulence model or the inappropriate turbulence boundary conditions applied at the interface.

For laminar flows, the fluid properties and boundary conditions are well defined. This motivates the present study to investigate more fundamentals of fluid mixing and the associated interface condensation process by a submerged laminar jet. For a typical cryogenic storage tank, the maximum liquid height to tank diameter ratio is about one and the mean-flow velocity is generally much larger than the turbulence at the interface. It is hoped that the study of laminar jet-induced mixing can provide some useful information for studying the turbulent jet-induced mixing in a typical cryogenic tank. In space, the effect of buoyancy force may be insignificant due to the low-gravity environment. Therefore, the fluid mixing in low-g may be equivalent to the mixing of constant-density liquid in normal gravity provided that the effect of free surface configuration is excluded. The present study is conducted for a normal-g environment with the neglecting of buoyancy force due to constant density. Numerical solutions are obtained by using a finite-difference method to solve nondimensional continuity, momentum and energy equations. Calculations encompass a wide range of the Prandtl numbers and jet Reynolds numbers. Detailed analyses are performed and simplified equations are generated to describe the effects of the above parameters on the condensation Nusselt and Stanton numbers which characterize the steady-state condensation process at the liquid-vapor interface.

\section{THE PHYSICAL PROBLEM CONSIDERED}

The physical system and the coordinates used to analyze the problem are shown in figure 1. A circular cylindrical tank of diameter $D$ contains liquid with a filling height $x_{s}$. An axisymmetric laminar jet with a velocity $u_{j}$ is discharged from the central part of the tank bottom toward the 1iquid-vapor interface. The liquid with same volume flow rate, $Q$, is withdrawn from the outer part of the tank bottom such that the liquid fill level is kept constant. The outflow area, Aout, is much larger than inflow jet area, $A_{j}$, (about 357:1) such that the outflow velocity is very small compared with the jet velocity. Both the central jet and outflow velocities are assumed uniform. The ratio of 
jet diameter, $d$, to tank diameter, $D$, is fixed at $1: 20$. The ratio of 1 iquid height to tank diameter, $x_{s} / D$, is set to be one.

The liquid-vapor interface is assumed to be flat (wave free) and shear free and at a constant saturation temperature, $T_{s}$. All the solid walls are insulated. The central jet is kept at a constant temperature, $T_{j}$, lower than the interface temperature. All the associated thermodynamics and transport properties are assumed constant since only small temperature variation is considered in the whole flow field. The outflow region is assumed to have zero temperature gradient.

The energy balance at the interface yields the following relation between condensation mass flux and heat transfer

$$
\bar{m}_{c} h_{f g} A_{s}=\int m_{c} h_{f g} d A_{s}=\int\left(k \frac{\partial T}{\partial x}\right)_{s} d A_{S}
$$

where $\overline{m_{c}}$ and $m_{c}$ are the average and local, respectively, condensation mass fluxes at the interface, $h_{f g}$ is the latent heat of condensation, and $A_{s}$ is the interface surface area. Experimentally, the steady-state condensation rate can be obtained based on the measurement of the temperature difference between outflow and inflow jet (ref. 5). The relation of energy balance for the whole system yields

$$
\bar{m}_{C} h_{f g} A_{s}=\frac{\rho Q C_{p}\left(T_{\text {out }}-T_{j}\right)}{\left(1+J a_{b}\right)}
$$

where $Q$ is the jet volume flow rate, Jab is the bulk Jacob number defined as $c_{p}\left(T_{s}-T_{\text {out }}\right) / h_{f g}$, and $T_{\text {out }}$ is the outflow temperature which is essentially uniform over the outflow area. Equations (1) and (2) can be used as a checkup of the numerical solutions. For commonly used cryogens such as hydrogen and nitrogen, Jab is usually much less than one. For calculation convenience, $\mathrm{Jab}$ is assumed zero in the present study. This assumption will be justified by the obtained solution later on.

A local condensation heat-transfer coefficient, $h_{c}$, and a local condensation Stanton number, St ${ }_{c}$, which describe the interfacial heat and mass transports can be defined as

$$
\begin{gathered}
h_{c}=\frac{m_{c} h_{f g}}{\left(T_{s}-T_{j}\right)} \\
s t_{c}=\frac{{ }^{h_{c}}}{\rho u_{j} C_{p}}
\end{gathered}
$$

It is noted that, instead of the bulk liquid temperature and the interface velocity, the jet temperature and the jet velocity, respectively, are used in equations ( 3 ) and (4). There are two reasons for these unusual definitions. First, the jet conditions are generally easy to control and measure so that the definitions in equations (3) and (4) may be more useful. Second, the difference between bulk temperature and interface temperature is very small in the present study so that $h_{c}$ will be very sensitive to the numerical error if $\left(T_{s}-T_{b}\right)$ is used in equation (3). For convenience, the average values of $h_{C}$ 
and $S t_{c}$ at the interface are generally used in the analysis and are expressed as

$$
\begin{gathered}
\bar{h}_{c}=\frac{\bar{m}_{c} h_{f g}}{\left(T_{s}-T_{j}\right)} \\
\overline{s t}_{c}=\frac{\overline{h_{c}}}{\rho u_{j} c_{p}}
\end{gathered}
$$

\section{MATHEMATICAL FORMULATION}

The jet-induced mixing considered in the present study is steady-state and incompressible with gravity acting in the vertical $-x$ direction. The governing equations for this axisymmetric laminar flow problem are:

$$
\begin{gathered}
\frac{\partial}{\partial x}(u)+\frac{\partial}{r \partial r}(r v)=0 \\
\frac{\partial}{\partial x}\left(u^{2}\right)+\frac{\partial}{r \partial r}(u r v)=-\frac{1}{\rho} \frac{\partial p}{\partial x}+g+\frac{\mu}{\rho} \frac{\partial^{2} u}{\partial x^{2}}+\frac{\mu}{\rho} \frac{\partial}{r \partial r}\left(r \frac{\partial u}{\partial r}\right) \\
\frac{\partial}{\partial x}(u v)+\frac{\partial}{r \partial r}\left(r v^{2}\right)=-\frac{1}{\rho} \frac{\partial p}{\partial r}-\frac{\mu}{\rho} \frac{v}{r^{2}}+\frac{\mu}{\rho} \frac{\partial^{2} v}{\partial x^{2}}+\frac{\mu}{\rho} \frac{\partial}{r \partial r}\left(r \frac{\partial v}{\partial r}\right) \\
\frac{\partial}{\partial x}(u T)+\frac{\partial}{r \partial r}(v r T)=\frac{k}{\rho C_{p}} \frac{\partial^{2} T}{\partial x^{2}}+\frac{k}{\rho C_{p}} \frac{\partial}{r} \frac{\partial r}{\partial r}\left(r \frac{\partial T}{\partial r}\right)
\end{gathered}
$$

The boundary conditions are applied for the system sketched in figure 1. At the centerline, the symmetric conditions are used:

$$
v=0, \quad \frac{\partial u}{\partial r}=\frac{\partial T}{\partial r}=0
$$

At the solid walls, the nonslip and adiabatic conditions are employed:

$$
u=v=0, \quad \frac{\partial T}{\partial x}=\frac{\partial T}{\partial r}=0
$$

The free surface is assumed to be flat (wave free) and shear free. The condensation-induced velocity at the interface, $u_{c}$, is equal to

$$
u_{C}=\frac{m_{C}}{\rho}
$$

and is assumed negligible compared with surface velocity. This assumption will also be justified by the obtained numerical solutions. The neglecting of 
condensation-induced velocity $u_{c}$ leads to the uncoupling between fluid dynamics and thermal calculations. Thus, the free surface boundary conditions for velocity are,

$$
u=0, \quad \frac{\partial v}{\partial x}=0
$$

The temperature of the interface is kept constant at $T_{S}$. For the jet inlet, the velocity and temperature are assumed to be uniform:

$$
u=u_{j}, \quad v=0, \quad T=T_{j}
$$

Liquid is withdrawn from the outer part of the tank bottom with the same volume flow rate as that of the injected liquid jet, so that the uniform withdrawing velocity can be obtained by $Q / A_{\text {out }}$. The conditions for radial velocity and temperature applied at this liquid-withdrawing plane are given by

$$
v=0, \quad \frac{\partial T}{\partial x}=0
$$

Nondimensionalized variables can be formed as

$$
x^{*}=\frac{x}{D}, \quad r^{*}=\frac{r}{D}, \quad u^{*}=\frac{u}{u_{j}}, \quad v^{*}=\frac{v}{u_{j}}, \quad p^{*}=\frac{p-p_{g}}{\rho u_{j}^{2}}, \quad T^{*}=\frac{T-T_{j}}{T_{s}-T_{j}}
$$

where, for convenience, the gravity term has been subtracted from the $x$-momentum equation by using the static equilibrium equation:

$$
\frac{\partial p_{g}}{\partial x}=-\rho g
$$

The dimensionless forms of the steady state governing equations are

$$
\begin{aligned}
& \frac{\partial u^{\star}}{\partial x^{\star}}+\frac{\partial r^{\star} v^{\star}}{r^{\star} \partial r^{\star}}=0 \\
& \frac{\partial u^{\star 2}}{\partial x^{\star}}+\frac{\partial u^{\star} r^{\star} v^{\star}}{r^{\star} \partial r^{\star}}=-\frac{\partial p^{\star}}{\partial x^{*}}+\frac{1}{B \operatorname{Re} j}\left[\frac{\partial^{2} u^{\star}}{\partial x^{\star}}+\frac{\partial}{r^{\star} \partial r^{\star}}\left(r^{\star} \frac{\partial u^{\star}}{\partial r^{\star}}\right)\right] \\
& \frac{\partial u^{*} v^{*}}{\partial x^{\star}}+\frac{\partial r^{\star} v^{\star}}{r^{\star} \partial r^{\star}}=-\frac{\partial p^{*}}{\partial r^{\star}}-\frac{1}{B \operatorname{Re} j} \frac{v^{*}}{r^{\star}}+\frac{1}{B \operatorname{Re} j}\left[\frac{\partial^{2} v^{*}}{\partial x^{*}}+\frac{\partial}{r^{\star} \partial r^{\star}}\left(r^{\star} \frac{\partial v^{\star}}{\partial r^{\star}}\right)\right] \\
& \frac{\partial u^{\star} T^{\star}}{\partial x^{\star}}+\frac{\partial r^{\star} v^{\star} T^{\star}}{r^{\star} \partial r^{\star}}=\frac{1}{B \operatorname{Re} j \operatorname{Pr}}\left[\frac{\partial^{2} T^{\star}}{\partial x^{\star 2}}+\frac{\partial}{r^{\star} \partial r^{\star}}\left(r^{\star} \frac{\partial T^{\star}}{\partial r^{\star}}\right)\right]
\end{aligned}
$$

The relevant parameters in the governing equations are the jet Reynolds number $\left(\operatorname{Re}_{j}\right)$, the Prandtl number $(\mathrm{Pr})$ and tank to jet diameter ratio (B):

$$
\operatorname{Re}_{j}=\frac{\rho u_{j} d}{\mu}, \quad \operatorname{Pr}=\frac{C_{p} \mu}{k}, \quad B=\frac{D}{d}
$$


The average condensation Nusselt number at the interface, $\overline{N u_{c}}$, is defined by

$$
\overline{N u}_{c}=\frac{\overline{h_{C}} D}{k}
$$

From equations (5) and ( 6 ), the average condensation Nusselt number and Stanton number can then be expressed as

$$
\begin{gathered}
\overline{N u_{c}}=8 \int\left(\frac{\partial T^{*}}{\partial x^{*}}\right)_{S} r^{*} d r^{*} \\
\overline{S t_{c}}=\frac{\overline{N u_{c}}}{B \operatorname{Re}_{j} \operatorname{Pr}}
\end{gathered}
$$

The corresponding boundary conditions then become:

At the centerline (symmetric conditions)

$$
v^{*}=0, \quad \frac{\partial u^{*}}{\partial r^{*}}=\frac{\partial T^{*}}{\partial r^{*}}=0
$$

At the solid walls (nonslip and adiabatic conditions)

$$
u^{*}=v^{*}=0, \quad \frac{\partial T^{*}}{\partial x^{*}}=\frac{\partial T^{*}}{\partial r^{*}}=0
$$

At the interface

$$
u^{*}=0, \quad \frac{\partial v^{*}}{\partial x^{*}}=0, \quad T^{*}=1
$$

At the jet inlet

$$
u^{*}=1, \quad v^{*}=0, \quad T^{*}=0
$$

At liquid-withdrawn plane

$$
u^{*}=\frac{-Q}{u_{j} A_{\text {out }}}, \quad v^{*}=0, \quad \frac{\partial T^{*}}{\partial x^{*}}=0
$$

The effects of jet Reynolds number $\left(\operatorname{Re}_{j}\right)$ on the dynamics of fluid mixing in the tank and the steady-state condensation rate at the liquid-vapor interface are investigated. Calculations will be performed with the jet Reynolds number ranging from 150 to 600 in which the flow is expected to be laminar according to the experiments of McNaughton and Sinclair (ref. 7). The Prandt) number ( $\mathrm{Pr}$ ) will be varied from 0.85 to 2.65 to represent various cryogens such as hydrogen and nitrogen so that the performance of fluid mixing can be evaluated. 


\section{NUMERICAL METHOD OF SOLUTIONS}

The above dimensionless forms of elliptic partial differential equations are numerically solved by a finite-difference method. The finite-difference equations are derived by integrating the differential equations over an elementary control volume surrounding a grid node appropriate for each dependent variable (ref. 8). A staggered grid system is used such that the scalar properties, $p$ and $T$, are stored midway between the $u$ and $v$ velocity grid nodes. The bounded skew hybrid differencing (BSHD) is incorporated for the convective terms (ref. 8) and the integrated source terms are linearized. Pressures are obtained from a predictor-corrector procedure of the Pressure Implicit Split Operator (PISO) method (ref. 9) which yields the pressure change needed to procure velocity changes to satisfy mass continuity. The governing finite-difference equations are solved iteratively by the ADI method with under relaxation until the solutions are converged.

Calculations are performed with a nonuniform grid distribution with concentration of the grid nodes in the centerline, near-wall, and near-interface regions where the gradients of flow properties are expected to be large. The nonuniform grid distribution in axial direction is generated by using an exponential function of Roberts' transformation (ref. 10) with stretching parameter equal to 1.02. In the radial direction, the scheme (ref. 11) with a constant ratio between two adjacent grid spacing is used. This ratio is set to be about 1.22 in the present study. To check grid dependency, the cases with 24 by 13 , 36 by 20,48 by 27,60 by 34 , and 72 by 41 nodes are run for jet Reynolds number $\left(R_{j}\right)$ equal to 600 and Prandt 1 number equal to 1.25 . Figures 2 and 3 show that there are only minor changes in $\left(\partial u^{*} / \partial r^{*}\right)$ distribution along the wall and $\left(\partial T^{*} / \partial x^{*}\right)$ distribution across the interface between grid distributions 60 by 34 and 72 by 41 . It is then decided to use 72 by 41 grid nodes for all the calculations in the present study. Calculations are performed on a CRAYXMP computer located at NASA Lewis Research Center. The convergent solutions are considered to be reached when the absolute value of (eq. (2) - eq. (1))/ (eq. (1)) is less than 0.003 and the maximum of absolute residual sums for each dependent variables is less than $10^{-6}$.

\section{RESULTS AND ANALYSIS}

A potential core length, $x_{p}$, is defined as the distance between the jet exit and the point where the jet centerline velocity begins to decrease. As shown in figure 4 , the potential core length is linearly increasing with jet Reynolds number and can be described by the following equation

$$
\frac{x_{p}}{d}=C_{1} R_{j}
$$

where $C_{1}$ is 0.0067 for the tankage considered in the present study. The conclusion of a linear relation between potential core length and jet Reynolds number is in agreement with the analysis of free jet in reference 12 . The centerline velocity, $u_{c t}^{*}$, decays slowly approaching the liquid-vapor interface. The velocity drops to zero abruptly at the interface with greater decreasing rate for a higher jet Reynolds number. The upstream effect of the existence of the interface is less for higher jet Reynolds numbers. It is noted that both the potential core length and the decaying rate of centerline velocity are less than those in reference 12 because of the difference between two physical flow 
systems considered resulting in different values of constant $C_{1}$. As the jet approaches the interface, an impingement region is created right underneath the interface. This region plays the most important role in the bulk-mixing process since the flow in this region removes the heat from the interface into the bulk liquid and induces the vapor condensation. The radial distribution of the free surface velocity, $v_{s}^{*}$, is shown in figure 5 . The $v_{s}^{*}$ increases from zero at the centerline to reach a peak shortly and then decreases approaching the side wall. For a higher jet Reynolds number, this peak velocity is larger and is located closer to the centerline. As expected, the average dimensionless surface velocity $\overline{v_{S}^{*}}$ is increasing with $R_{j}$ and is approximately proportional to $\mathrm{Re}_{\mathrm{j}} / 2$ :

$$
\overline{v_{s}^{*}}=\frac{\overline{v_{s}}}{u_{j}}=C_{2} \operatorname{Re}_{j}^{1 / 2}
$$

where $C_{2}$ is 0.0134 in the present study. Consequently, the average interface radial velocity is approximately increasing with $\mathrm{u}_{j}^{3 / 2}$.

The existence of liquid-vapor interface and tank side walls and the withdrawing of the liquid from the outer part of the tank bottom enhance the flow circulation in the tank. Figure 6 shows the velocity gradient distribution at the side wall owing to the liquid flowing toward the tank bottom. Because the backward axial velocity is very small the wall shear is negligible at most of the tank side walls except the region near the interface. The value of the maximum wall shear is higher and is located closer to the interface for jets with higher Reynolds number. The distance of the location of maximum wall shear from the interface is observed to be approximately the same as the thickness of the impingement region. From figure 6 , the radial boundary layer thickness appears to reach a value of about $0.025 \mathrm{D}$ (i.e., about a value of 0.5 d) as the jet Reynolds number is increased. This finding is in agreement with that for a turbulent jet in reference 2 .

For Prandtl number $\operatorname{Pr}=1.25$, the axial temperature distribution at the centerline, $R=0$, as a function of jet Reynolds number is shown in figure 7 . The centerline temperature increases slowly approaching the interface with higher increasing rate for a smaller jet Reynolds number. Consequently, in the region near the interface, higher jet Reynolds numbers yield much higher temperature gradients to approach the interface temperature. The radial distribution of temperature gradient at the interface is given in figure 8 . As observed, a high jet Reynolds number flow induces more interface condensation. Most of the mass condensation occurs in the central part of the interface for the ratio of jet diameter to tank diameter equal to 0.05 considered in the present study. It is expected that if the jet nozzle to tank diameter ratio is small enough, the difference of interface configuration between low-g and normal-g will not have significant effect on the interface condensation. Therefore, as mentioned in the introduction section, the fluid mixing processes of low-g and normal-g can be equivalent if the buoyancy force is neglected. Calculations were also performed for Prandt] numbers varied from 0.85 to 2.65 with various jet Reynolds numbers. The effect of Prandtl number on the distributions of centerline temperature and interface temperature gradient was found to be similar to that of jet Reynolds number as shown in figures 7 and 8 . Higher Prandtl number will yield larger condensation heat flux over the interface and thinner thickness of thermal layer underneath the interface. It is 
noted that for higher jet Reynolds numbers and Prandtl numbers, the maximum of temperature gradient at the interface is slightly shifted away from the centerline due to the effect of significant surface motion. The radial distribution of temperature at $x^{*}=0.97$ is shown in figure 9 . The higher jet Reynolds number yields lower flow temperature in the central jet region, approaching bulk liquid temperature with higher increasing rate at the edge of jet region, and results in a thinner thermal layer underneath the interface.

The dimensionless condensation-induced velocity at the surface, $u_{c}^{*}$, can be calculated by

$$
u_{c}^{*}=\frac{u_{c}}{u_{j}}=\frac{\left(\partial T^{*} / \partial x^{*}\right)_{s} J a_{j}}{\operatorname{Pr} \operatorname{Re}_{j} B}
$$

where the jet Jacob number is defined as $C_{p}\left(T_{s}-T_{j}\right) / h_{f g}$. The maximum of $u_{c}^{*}$ is located at the centerline. For liquid hydrogen at a saturation pressure of 30 psia with $2{ }^{\circ} R$ of jet subcooling, the maximum values of $u_{c}^{*}$ for $R_{j}=600$ and 150 are equal to 0.0011 and 0.0009 , respectively. From figure 5 , it is observed that $u_{c}^{*}$ is negligibly small compared with surface velocity $v_{s}^{*}$ except for the very small stagnation region. The temperature difference between the bulk liquid and the interface is much smaller than that between the jet and the interface. For $\left(T_{s}-T_{j}\right)$ equal to $2^{\circ} \mathrm{R}$, ( $T_{s}-T_{\text {out }}$ ) is approximately equal to $0.06^{\circ} \mathrm{R}$ and the corresponding bulk Jacob number, Jab, is equal to 0.001 . It can then be justified that the assumption of neglecting of the condensation-induced velocity, $u_{c}$, and bulk Jacob number, Jab, in the present study is acceptable.

The correlation for the average condensation Nusselt number is assumed to be of the form:

$$
\overline{N u_{C}}=C_{3} \operatorname{Re}_{j}^{m} \operatorname{Pr}^{n}
$$

Based on the obtained numerical solution, the values of $c_{3}, m$, and $n$ are approximately equal to $0.049,1.0$, and 0.97 , respectively. As shown in figures 10 and 11 , the average condensation Nusselt number is linearly proportional to jet Reynolds number and nearly a linear function of Prandt l number. It is noted that the constant $n$ is actually a weak function of Prandtl number itself and is decreasing from 1.0 for $\mathrm{Pr}=0.85$ to 0.95 for $\mathrm{Pr}=2.65$. The value of $n$ is expected to slightly decrease as the Prandtl number keeps increasing. Equation (10) also implies that the condensation mass flux is linearly increasing with jet Reynolds number which is in agreement with the data for turbulent jet in reference 3 . From equations (9) and (10), the correlation for average condensation Stanton number can be expressed as

$$
\overline{S t_{C}}=C_{4} \operatorname{Pr}^{n-1}=C_{4} \operatorname{Pr}-0.03
$$

where $C_{4}$ is equal to 0.00245 . The average condensation Stanton number is a weak function of Prandtl number only and is independent of jet Reynolds number. In the present study, only the jet Reynolds number and the Prandtl number are investigated and the other relevant tankage system parameters such as $B(=D / d), x_{s} / D$, and $A_{\text {out }} / A_{j}$ are specified. Therefore, it is expected that the correlation constants, $C_{1}, C_{2}, C_{3}$ and $C_{4}$, obtained from the above analysis need to be modified if a different tankage system is considered. 
From equations (2), (5), and (6), the average condensation Stanton number for $J_{a b}=0$ can be expressed as

$$
\overline{s t_{c}}=\left(\frac{T_{\text {out }}-T_{j}}{T_{s}-T_{j}}\right)\left(\frac{d}{D}\right)^{2}
$$

If the outflow temperature Tout approaches $T_{S}$, equation (12) will become

$$
\overline{S t_{c}} \approx\left(\frac{d}{D}\right)^{2}
$$

It implies that $\overline{s t_{c}}$ is nearly a constant and is simply determined by system geometry provided that the flow conditions can yield Tout close to $T_{5}$. The physical phenomenon behind equation (13) is that the cold jet simply enters, sweeps by the interface, and receives as much heat as it can absorb, reaching saturation temperature and exiting. Equation (13) gives an upper bound for $\overline{S t_{c}}$ based on an energy balance with the outflow liquid at its maximum possible temperature. In the present computation, $d / D=1 / 20$, and equation (13) gives

$$
\overline{S t_{C}}=0.0025
$$

which is in excellent agreement with the numerical correlation shown in equation (11). It can be concluded that for laminar operating conditions with a jet Reynolds number up to 600 , the injected liquid reaches essentially interface saturation temperature before exiting (at least for a tankage system of $d / D=1 / 20$ ), and the total condensation rate is controlled by the jet volume flow rate rather than by the condensation heat-transfer coefficient at the interface.

\section{CONCLUSIONS}

The nondimensional form of steady state continuity, momentum, and energy equations has been solved by a finite-difference method. Numerical solutions were obtained for a laminar jet-induced fluid mixing in a cryogenic tank system shown in figure 1. The temperature variation in the whole flow field was small so that the thermodynamics and transport properties were assumed constant. The effect of buoyancy force was neglected. The interface was assumed to be flat (wave free) and shear free. The condensation-induced velocity at the interface and the associated bulk and jet Jacob numbers were assumed zero. Calculations were performed for jet Reynolds numbers ranging from 150 to 600 and Prandtl numbers ranging from 0.85 to 2.65. Based on the tankage system considered in the present study, it is concluded that:

(1) The ratio of average radial interface velocity to jet velocity is approximately proportional to $\mathrm{Re}_{j} / 2$ and the average interface radial velocity is approximately proportional to $u_{j}^{3 / 2}$. The thickness of the impingement region layer underneath the interface is decreasing with increasing jet Reynolds number and appears to approach a value of one-half of the jet diameter as jet Reynclds number is further increased. 
(2) If the jet nozzle to tank diameter ratio is small enough, most of the vapor condensation will occur at the central part of the interface and the effect of the difference of interface configuration between low-g and normal-g on the interface condensation will be insignificant.

(3) The condensation Nusselt number and then the condensation mass flux are linearly increasing with jet Reynolds number. The condensation Stanton number is independent of jet Reynolds number and is proportional to $\operatorname{Pr} l-n$ with $n$ approximately equal to 0.97 for Prandtl numbers ranging from 0.85 to 2.65. The value of the exponent $n$ is slightly decreasing with increasing Prandtl number. The thermal-layer thickness underneath the interface is thinner for higher values of jet Reynolds number and Prandtl number.

(4) For laminar operating conditions with a jet Reynolds number up to 600 , the injected liquid reaches essentially interface saturation temperature before exiting (at least for a tankage system with $x_{s} / D=1$ and $d / D=1 / 20$ ), and the interface condensation rate is jet volume flow limited.

\section{REFERENCES}

1. Aydelott, J.C., Carney, M.J., and Hochstein, J.I., "NASA Lewis Research Center Low-Gravity Fluid Management Technology Program," NASA TM-87145, 1985.

2. Poth, L.J., and Van Hook, J.R., "Control of the Thermodynamic State of Space-Stored Cryogens by Jet Mixing," Journal of Spacecraft and Rockets, Vol. 9, No. 5, May 1972, pp. 332-336.

3. Thomas, R.M., "Condensation of Steam on Water in Turbulent Motion," International Journal of Multiphase Flow, Vol. 5, No. 1, Feb. 1979, pp. $1-15$.

4. Dominick, S.M., "Mixing Induced Condensation Inside Propellant Tanks," AIAA Paper 84-0514, Jan. 1984.

5. Sonin, A.A., Shimko, M.A., and Chun, J.H., "Vapor Condensation onto a Turbulent Liquid - I. The Steady Condensation Rate as a Function of Liquid-Side Turbulence," International Journal of Heat Mass Transfer, Vol. 29, No. 9, Sept. 1986, pp. 1319-1332.

6. Hasan, M.M. and Lin, C.-S., "Axisymmetric Confined Turbulent Jet Directed Towards the Liquid Surface From Below," AIAA Paper 89-0172, Jan. 1989, (NASA TM-101409.)

7. McNaughton, K.J. and Sinclair, C.G., "Submerged Jets in Short Cylindrical Flow Vessels," Journal of Fluids Mechanics, Vol. 25, Part 2, 1966, pp. 367-375.

8. Syed, S.A., Chiappetta, L.M., and Gosman, A.D., "Error Reduction Program," PWA-5928-25, Pratt and Whitney Aircraft Co., East Hartford, CT, 1985, NASA CR-174776. 
9. Issa, R.I., "Solution of the Implicitly Discretised Fluid Flow Equations by Operator-Splitting," Journal of Computational Physics, Vol. 62, No. 1 , Jan. 1986, pp. 40-65.

10. Roberts, G.0., "Computational Meshes for Boundary Layer Problems," Proceedings of the Second International Conference on Numerical Methods in Fluid Dynamics, Lecture Notes in Physics, Vol. 8, M. Holt, ed., Springer-Verlag, New York, 1971, pp. 171-177.

11. Cebeci, T., and Smith, A.M.O., "Analysis of Turbulent Boundary Layers," Academic, New York, 1974.

12. Gauntner, J.W., Livingood, J.N.B., and Hrycak, P., "Survey of Literature on Flow Characteristics of a Single Turbulent Jet Impinging on a Flat Plate," NASA TN D-5652, 1970. 


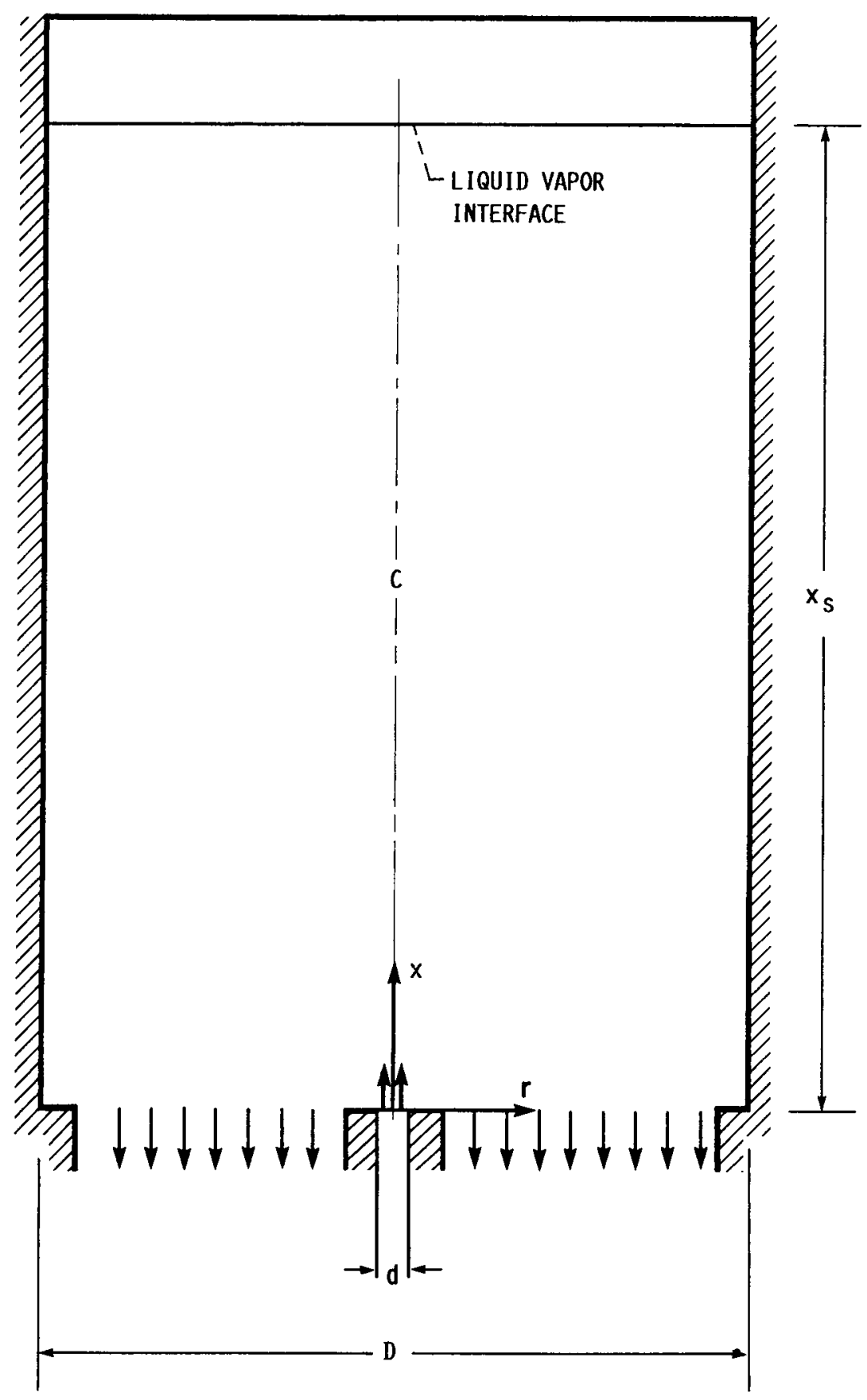

FIGURE 1. - PHYSICAL SYSTEM AND COORDINATES. 


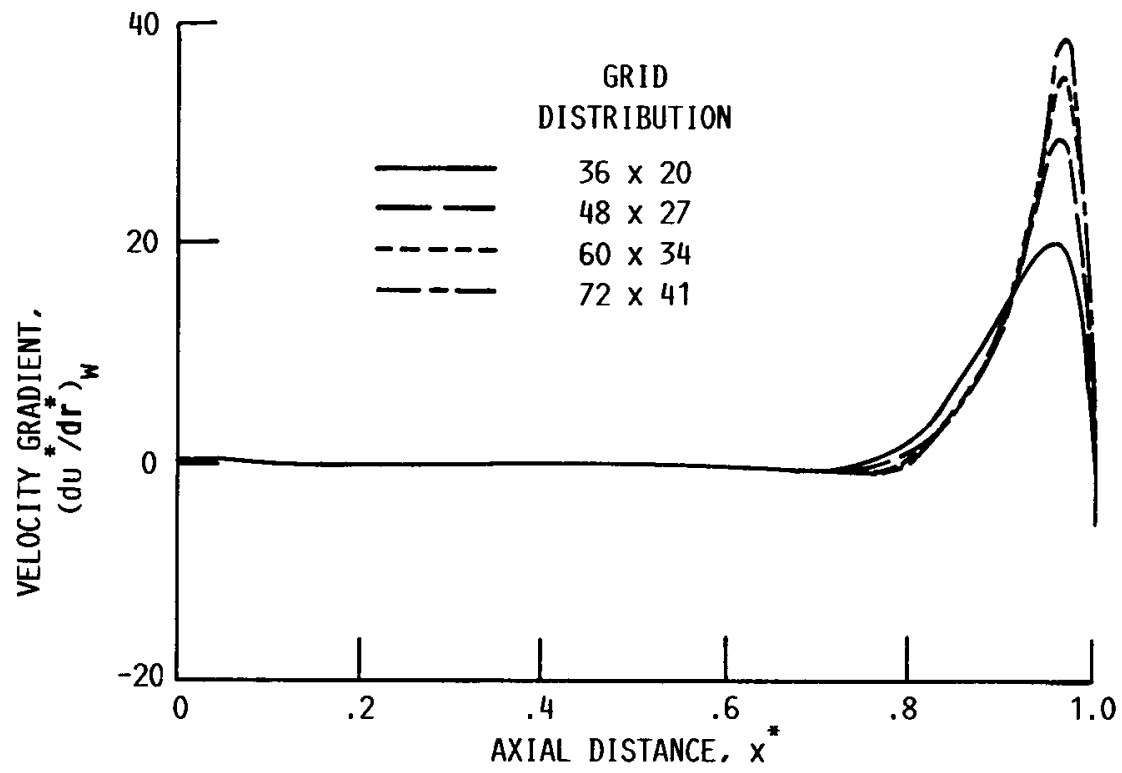

FIGURE 2. - DISTRIBUTION OF DIMENSIONLESS VELOCITY GRADIENT AT THE TANK SIDE WALL.

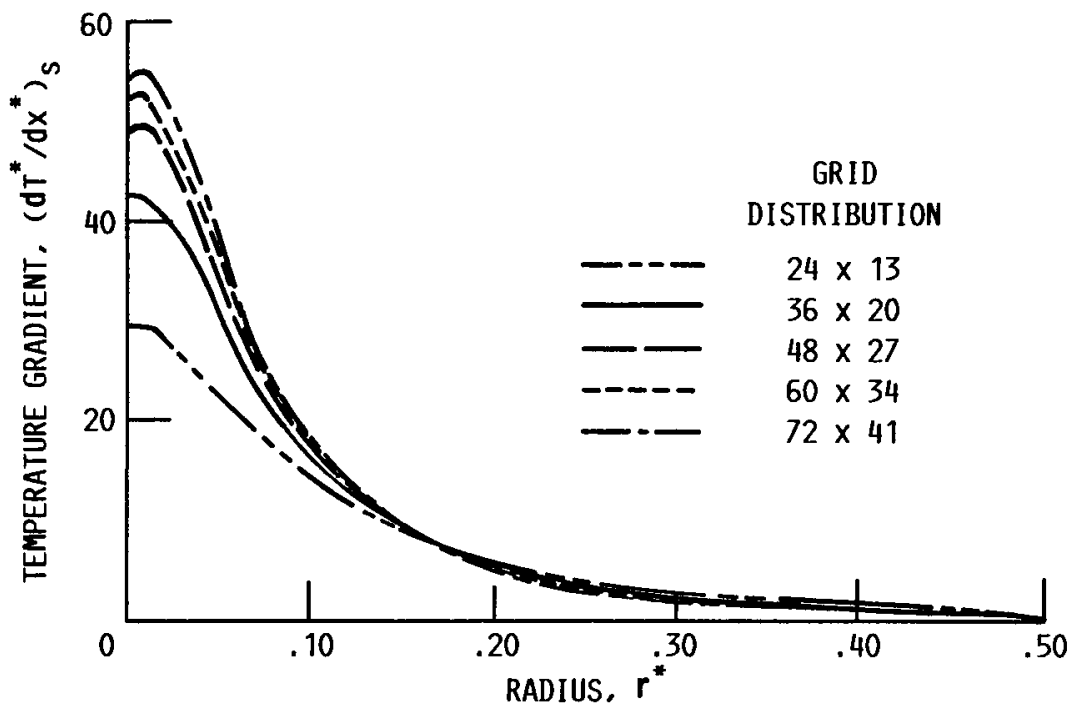

FIGURE 3. - RADIAL DISTRIBUTION OF DIMENSIONLESS TEMPERATURE GRADIENT AT THE INTERFACE. 


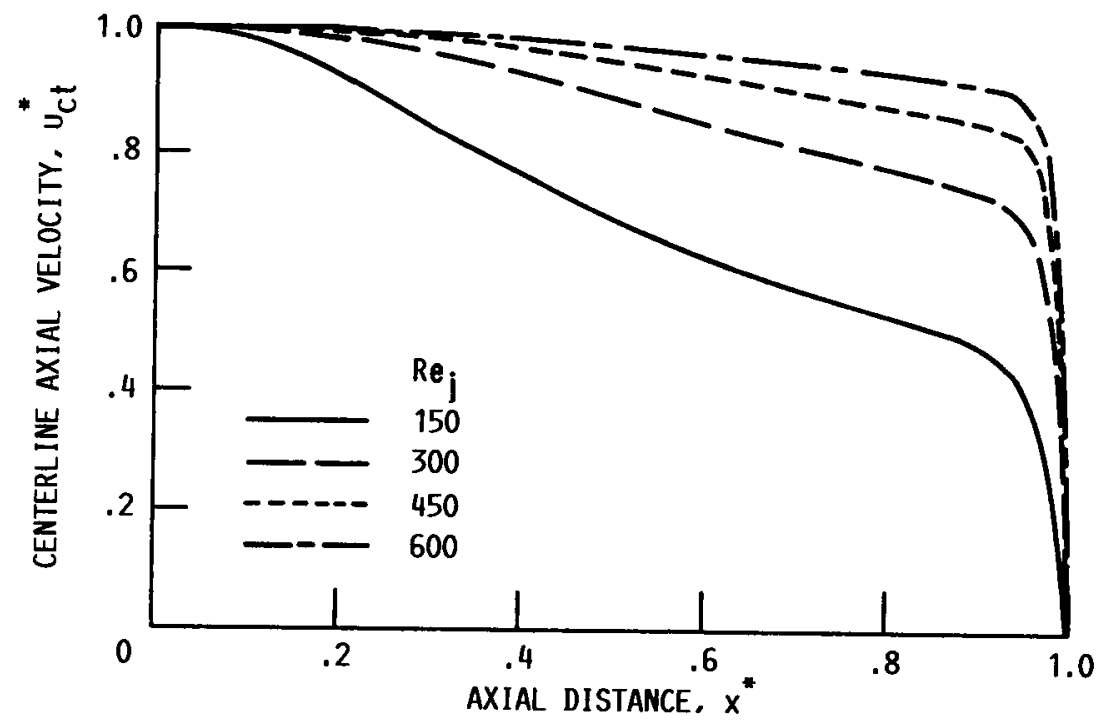

FIGURE 4. - DISTRIBUTION OF DIMENSIONLESS AXIAL VELOCITY ALONG THE CENTERLINE.

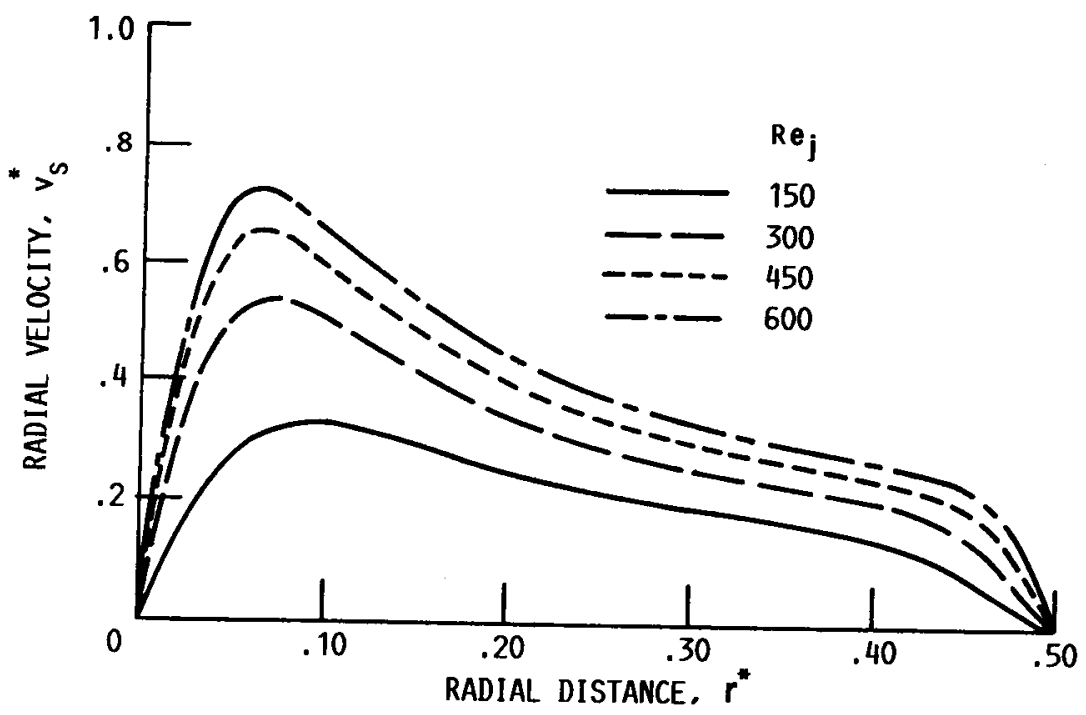

FIGURE 5. - DISTRIBUTION OF DIMENSIONLESS RADIAL VELOCITY AT THE INTERFACE. 


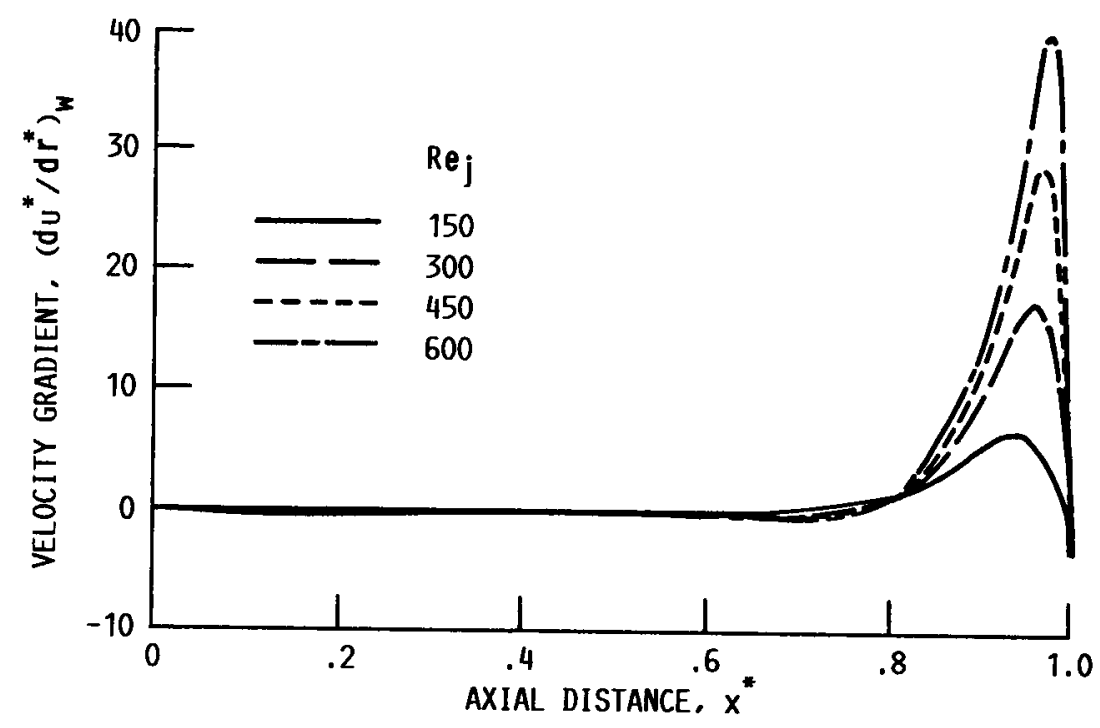

FIGURE 6. - DISTRIBUTION OF DIMENSIONLESS AXIAL VELOCITY GRADIENT ALONG THE TANK SIDE WALL.

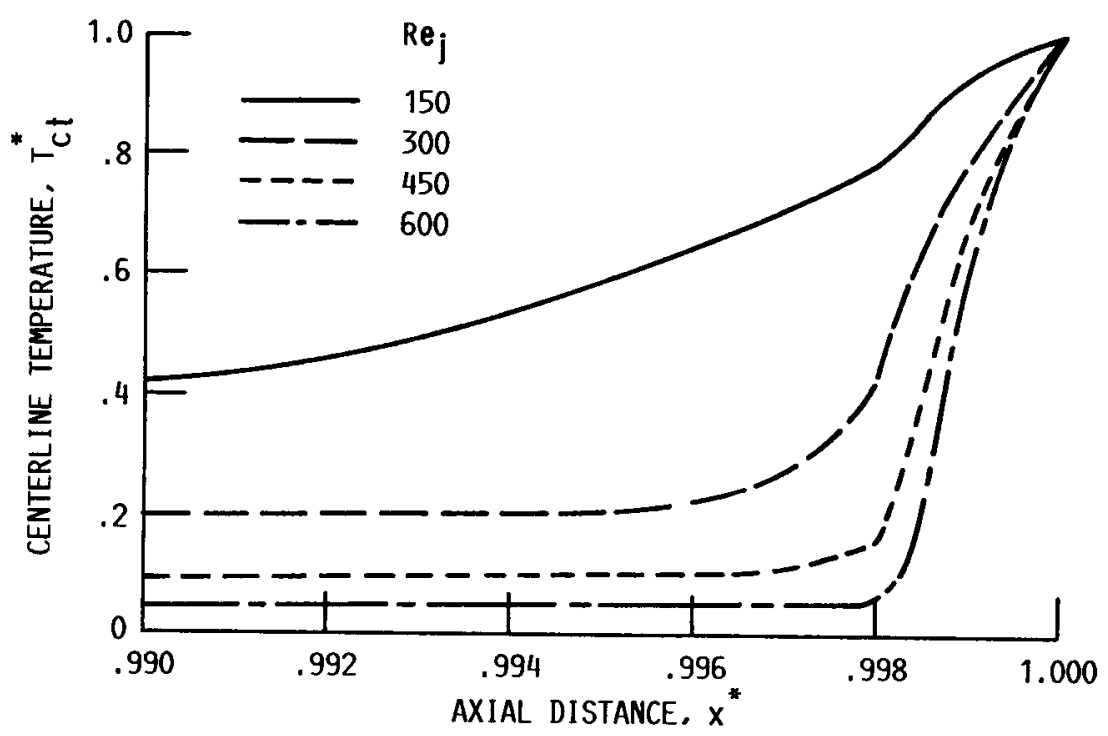

FIGURE 7.- DISTRIBUTION OF DIMENSIONLESS TEMPERATURE ALONG THE CENTERLINE FOR $\mathrm{Pr}=1.25$. 


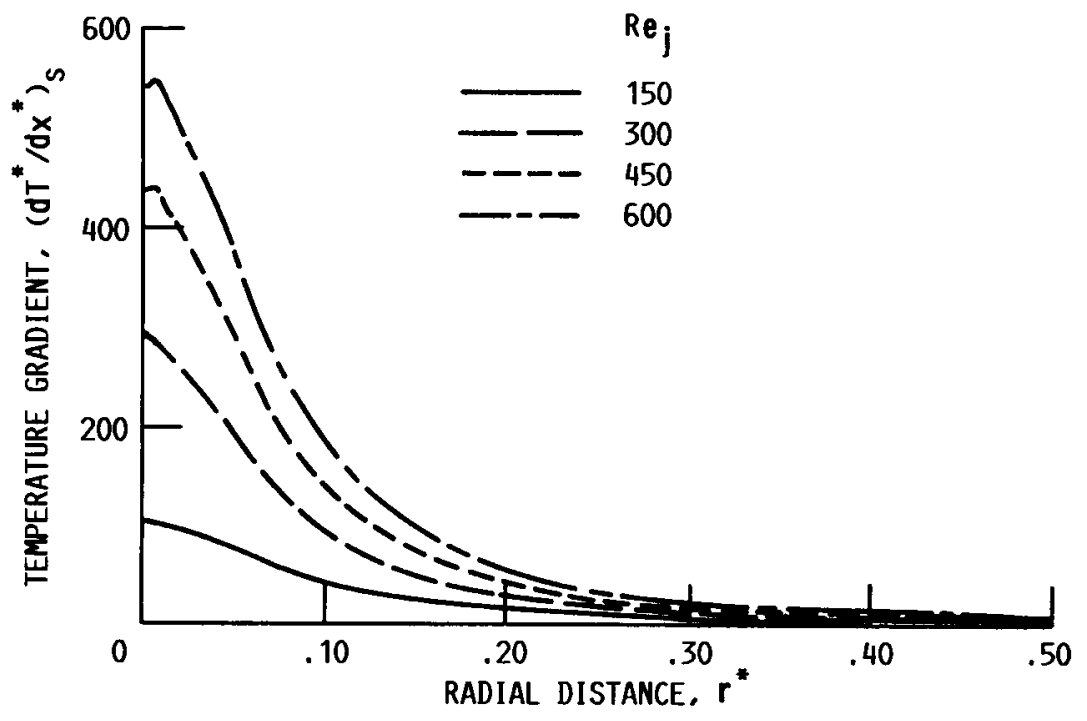

FIGURE 8. - RADIAL DISTRIBUTION OF DIMENSIONLESS TEMPERATURE GRADIENT AT THE INTERFACE FOR $\mathrm{Pr}=1.25$.

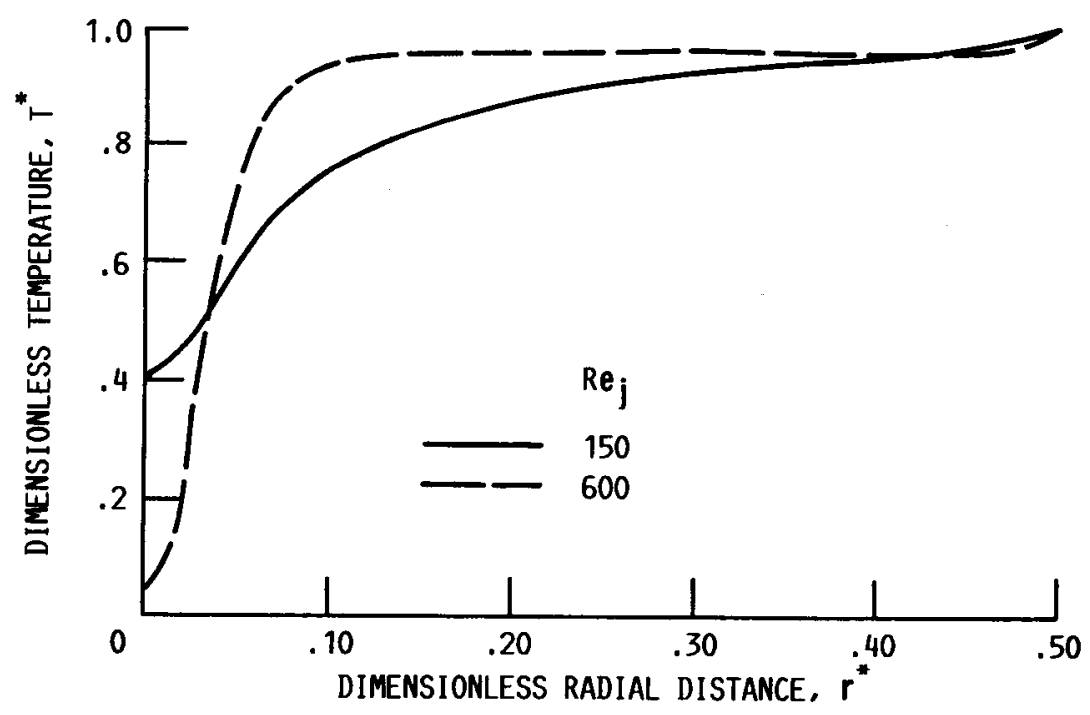

FIGURE 9. - RADIAL DISTRIBUTION OF TEMPERATURE AT $x^{*}=0.97$ AS A FUNCTION OF REYNOLDS NUMBER FOR Pr $=1.25$. 


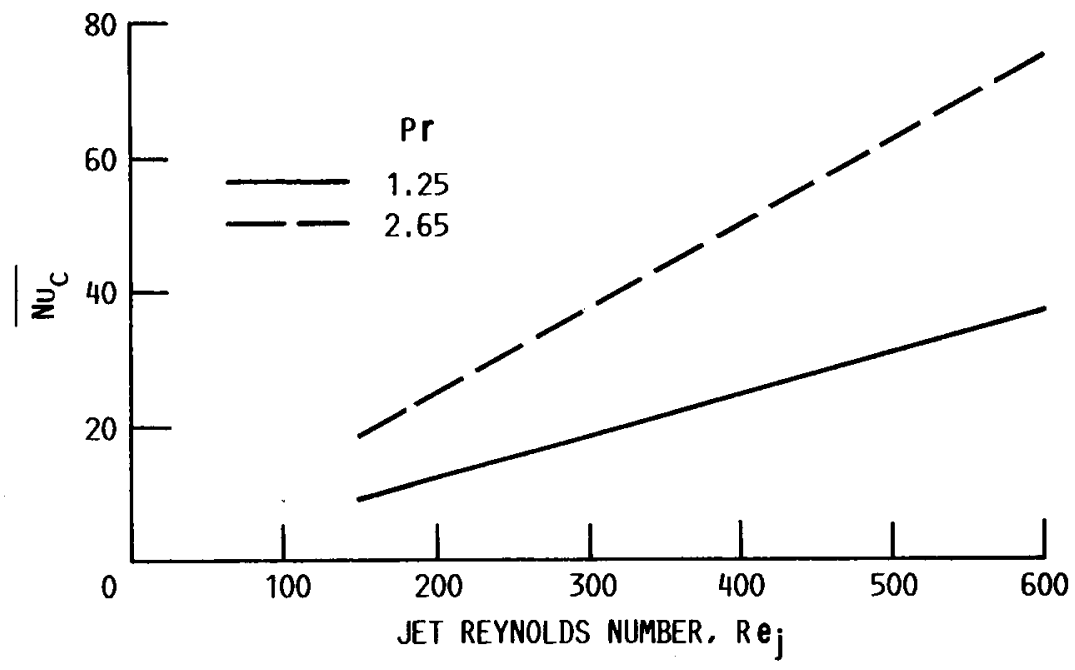

FIGURE 10. - AVERAGE CONDENSATION NUSSELT NUMBER AS A FUNCTION OF JET REYNOLDS NUMBER.

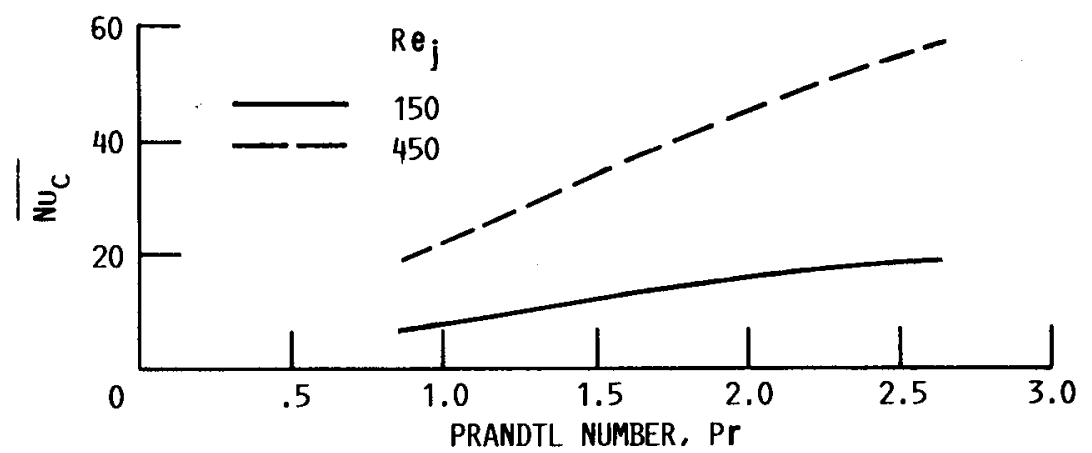

FIGURE 11. - AVERAGE CONDENSATION NUSSELT NUMBER AS FUNCTION OF PRANDTL NUMBER. 


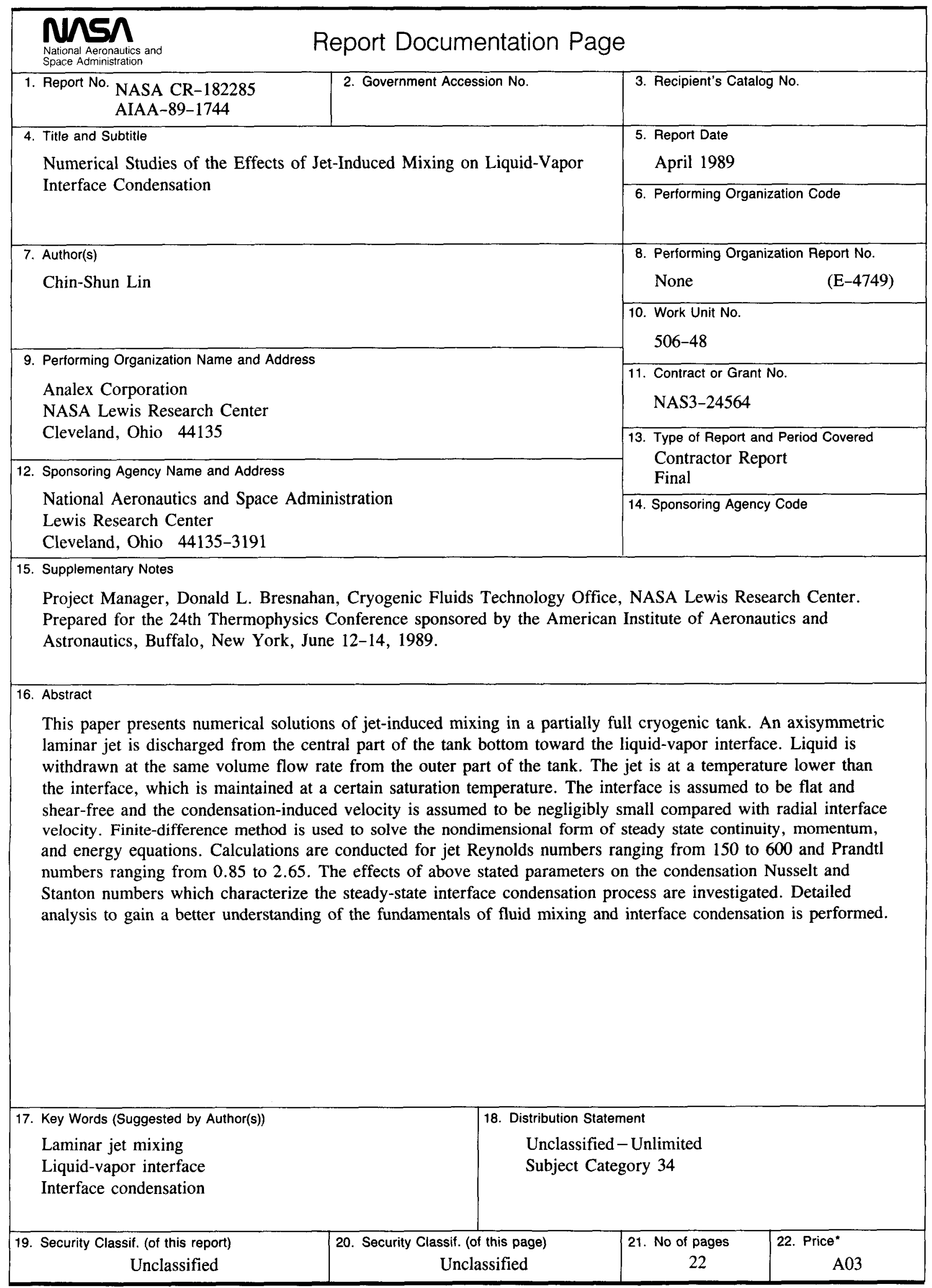

